\title{
Abordajes para cirugía de tercio medio facial. Algunas alternativas
}

\author{
Approaches for midface surgery. Some alternatives
}

Recibido: 2018/01/21. Aceptado: 2018/02/15. Publicado: 2018/03/15

\section{Evelyn Olalla López 1 \\ Patricio Unda Jaramillo ${ }^{2}$ \\ Rómulo Tamayo Clavijo ${ }^{3}$ \\ Jimmy Casares Tamayo ${ }^{4}$}

1 Universidad San Francisco de Quito. Colegio de Ciencias de la Salud, Escuela de Odontología, Clínica Odontológica, Campus Cumbayá, oficina CO 106, Casilla postal 17-1200-841, Quito-Ecuador.

Correo electrónico: evelynolalla@hotmail.com

2 Hospital Militar de las Fuerzas Armadas Quito HE-1, Servicio de Cirugía Oral y Maxilofacial. Queseras del Medio s/n y Av. Gran Colombia. Quito-Ecuador.

Correo electrónico: patundaja@hotmail.com

${ }^{3}$ Hospital Militar de las Fuerzas Armadas Quito HE-1, Servicio de Otorrinolaringología. Queseras del Medio s/n y Av. Gran Colombia. Quito-Ecuador.

Correo electrónico: drtamayo.orl@gmail.com

${ }^{4}$ Hospital Militar de las Fuerzas Armadas Quito HE-1, Servicio de Oncología. Queseras del Medio s/n y Av. Gran Colombia. Quito-Ecuador.

Correo electrónico: jirocas@yahoo.es 


\section{Resumen}

El tercio medio facial es susceptible a sufrir diferentes alteraciones o problemas como traumatismos, deformidades, fisuras, o patologías, que requieren ser resueltos quirúrgicamente. Para ello se han diseñado y se usan algunos abordajes que resultan en diferentes grados de variedad y tipo.

Se presentan dos alternativas útiles para abordajes de cirugías de tercio medio facial como son el Degloving Centro Facial y abordaje de Weber Ferguson, a manera de un estudio aplicado, comparativo, clínico, trasversal, retrospectivo y abierto realizado en el Hospital Militar HE-1 de Quito con buenos resultados a largo plazo. A través de este artículo puede conocerse las aplicaciones, contraindicaciones, ventajas y desventajas de estos abordajes en cirugía de tercio medio facial, además de una alternativa para que el cirujano maxilofacial pueda abordar las diferentes alteraciones que puedan presentarse a este nivel.

Palabras clave: abordajes, tercio medio facial, Degloving centro facial, Weber Ferguson

\section{Abstract}

The middle facial third is susceptible to suffer different alterations or problems such as trauma, deformities, clefts, or pathologies, which require being surgically resolved. For this purpose, some approaches have been designed and used that result in different degrees of variety and type.

We present two useful alternatives for approaches of middle facial third surgeries such as the Center Facial Degloving and Weber Ferguson approach, as an applied, comparative, clinical transverse, retrospective, and open study performed at the HE-1 Military Hospital of Quito with good results a long term. The objective of this article was present the application, contraindications, advantages and disadvantages of this approaches in surgery of midface, and provide an alternative to the maxillofacial surgeon, so they can approach the different alterations that may occur at this level.

Keywords: approaches, middle facial third, facial center Degloving, Weber Ferguson. 


\section{Introducción}

El tercio medio facial corresponde al aérea de la cara que se encuentra entre el tercio superior o frontal que es inmóvil y de fuerte resistencia estructural que protege y aloja al cerebro, y el tercio inferior mandibular, móvil e igualmente resistente ${ }^{1}$.

El tercio medio facial comprende estructuras más susceptibles en comparación a sus otros dos tercios faciales, aloja estructuras de la vía aérea superior un tanto más débiles con huesos que se asocian formando complejos que le dan una cierta consistencia de fortaleza. Sin embargo, el tercio medio facial es susceptible a alteraciones o problemas donde podemos enumerar, sin orden en particular, a traumatismos faciales como fracturas tipo Lefort II o Lefort III, fracturas orbito cigomáticas, fracturas NOE, entre otras; deformidades faciales como hipoplasia maxilar, fisuras 0-14 de Tessier; o, procesos patológicos oncológicos o neoplásicos lo que hace que cualesquiera de ellos deban solucionarse quirúrgicamente.

Desde hace muchos años, se han usado dos abordajes con diferente aplicación según cada caso, estos son el abordaje Degloving Centro Facial por vía intraoral con manejo casi exclusivo para oncología y procedimientos de otorrinolaringología, y otro abordaje, de Weber Ferguson que usa la vía extraoral. Sin embargo, la literatura también sugiere el uso de estos abordajes en el tratamiento de fracturas faciales, deformidades postraumáticas y reconstrucción facial ${ }^{2}$.

Se recomienda el uso del abordaje extraoral (Weber Ferguson) para casos de cirugía de tercio medio facial, cuando el traumatismo o patología va más allá del reborde infraorbitario por arriba o más allá de la región malar por fuera, esta técnica expone las fosas nasales, maxilar, etmoides, orbita, y la cara inferior de la base del cráneo; mientras que el abordaje intraoral (Degloving), se usa para casos donde el procedimiento no exceda las áreas antes mencionadas para el otro abordaje, pues este permite una buena exposición de las cavidades nasales, tercio medio de la cara y base del cráneo en su porción central ${ }^{3}$.

El abordaje Degloving intraoral está indicado para procedimientos de corrección de tercio medio, como son trauma facial (fracturas naso-orbitoetmoidales), resecciones oncológicas (maxilectomia), patologías (angiofibromas, enfermedad fibroósea), reparación de perforaciones septales amplias, osteotomías del tercio medio facial, injertos óseos en el tercio medio facial para la reconstrucción del contorno facial. Mientras que el abordaje Weber Ferguson se utiliza principalmente para la realización de la maxilectomia pura o que incluya el piso de la órbita, así como acceso tipo Lefort I, con seguridad y mínima morbilidad, sin cicatrices externas $\mathbf{4 , 5 , 6}$.

El objetivo de este artículo fue presentar la aplicación, contraindicaciones, ventajas y desventajas en de estos abordajes en cirugía de tercio medio facial, y además proporciona una alternativa más, para que el cirujano maxilofacial pueda abordar las diferentes alteraciones que puedan presentarse a este nivel.

\section{Caso 1}

Se presenta un primer caso; de un paciente masculino que acudió a la consulta de Oncología del Hospital de Especialidades HE-1 de las Fuerzas Armadas, con diagnóstico de estesioneuroblastoma y es referido para manejo multidisciplinario con Otorrinolaringología y Cirugía Maxilofacial.

El abordaje Weber Ferguson inicio con la incisión labial con bisturí \# 10 sobre la línea media del bermellón previamente marcada, la incisión subnasal se realiza sobre la base de la columella nasal y a 1 o $2 \mathrm{~mm}$ alrededor del ala nasal, se continua con la incisión nasal lateral entre la mejilla y nariz; este abordaje tiene algunas 

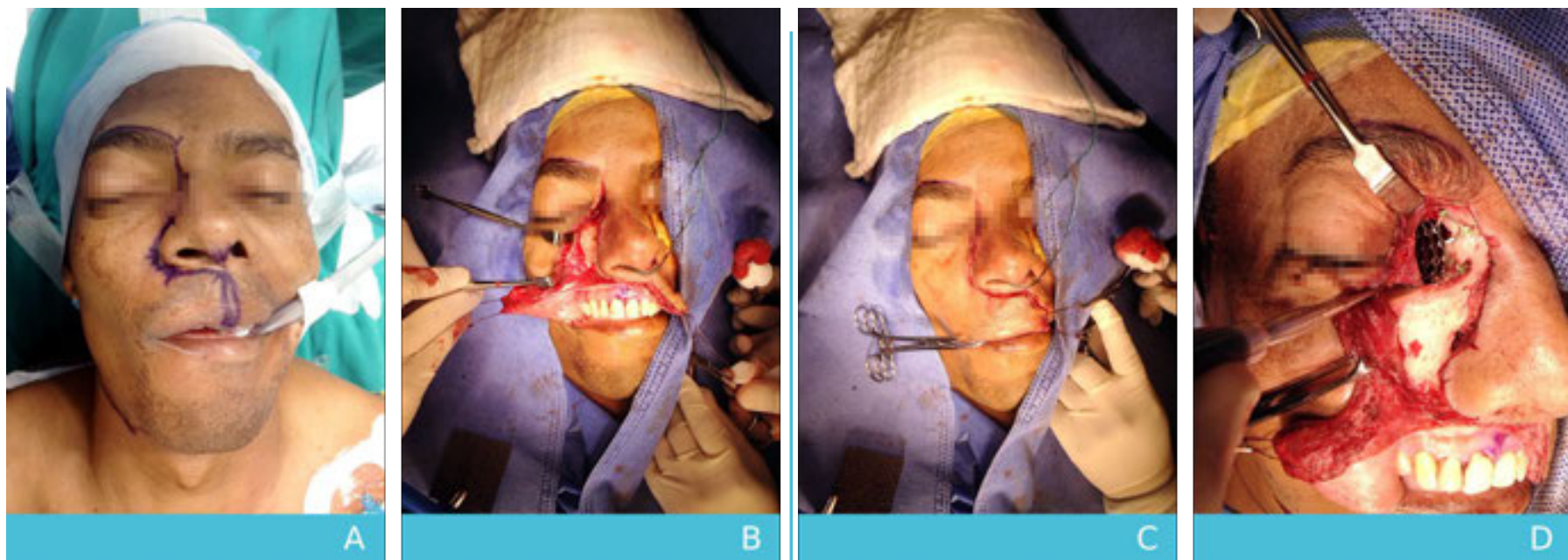

FIGURA 1. A: MARCAJE DE INCISIÓN WEBER FERGUSON. B: INCISIÓN COMPLETA. C: DISECCIÓN Y LEVANTAMIENTO DE COLGAJO. D: RESECCIÓN DE TUMOR Y RECONSTRUCCIÓN DE PARED MEDIAL ORBITA DERECHA CON MALLA DE OSTEOSÍNTESIS.

modificaciones, como la de Dieffenbach si es extendida lateralmente hacia abajo del párpado inferior en uno de los pliegues, de Lynch si es extendida superiormente hacia la ceja o de Borle si la incisión es extendida desde el canto lateral del ojo a la región temporal en forma de un signo de interrogación. La incisión intraoral se realiza a lo largo del proceso alveolar a nivel de la mucosa vestibular o bordes cervicales de los dientes. Se continuó con la elevación del colgajo de la cara en su porción supra o sub-perióstica según los requerimientos del procedimiento $\mathbf{1 , 3 , 4}$.

La incisión expone el campo quirúrgico y permitió la resección completa del tumor y reconstrucción de la pared medial de orbita derecha con una malla de titanio. Figura 1.

\section{Caso 2}

Se presenta un segundo caso; de un paciente masculino que acude a consulta de Cirugía Maxilofacial del Hospital de Especialidades HE-1 de las Fuerzas Armadas; por secuela de trauma facial (fractura de complejo orbito cigomático maxilar) tratada anteriormente por dos ocasiones en otra casa de salud con abordajes extra orales. Por lo que se planifico en conjunto con el Servicio de ORL abordaje alterno para resolución de la secuela.
El abordaje Deglovin centro facial, combina incisiones vestibulares con incisiones endonasales; se realizan tres incisiones intranasales que se conectan entre sí; la incisión intercartilaginosa bilateral, transfixión completa y apertura piriforme bilateral que permiten el acceso al dorso y base nasal, estas incisiones son hechas a través de la mucosa, submucosa, tejido aponeurótico y pericondrio, la disección subpericóndrica cortante con un bisturí o una disección roma con tijeras de bordes afilados libera los tejidos blandos del cartílago lateral superior. Se continua con la incisión vestíbulo maxilar en fondo de surco y una disección subperiostica exponiendo la cara anterior del maxilar y cigoma. La disección sub-periostica a lo largo de las porciones superiores de la apertura piriforme, conectaran dicha disección con la parte superior de los cartílagos laterales superiores de la nariz y huesos nasales. La elevación del labio y la columella con un gancho de piel permite que la punta nasal sea despegada del septum nasal y cartílagos laterales superiores, exponiendo superiormente hasta la línea de sutura frontonasal, el reborde infraorbitario y lateralmente, hasta el proceso cigomático $\mathbf{4 , 6}$. 

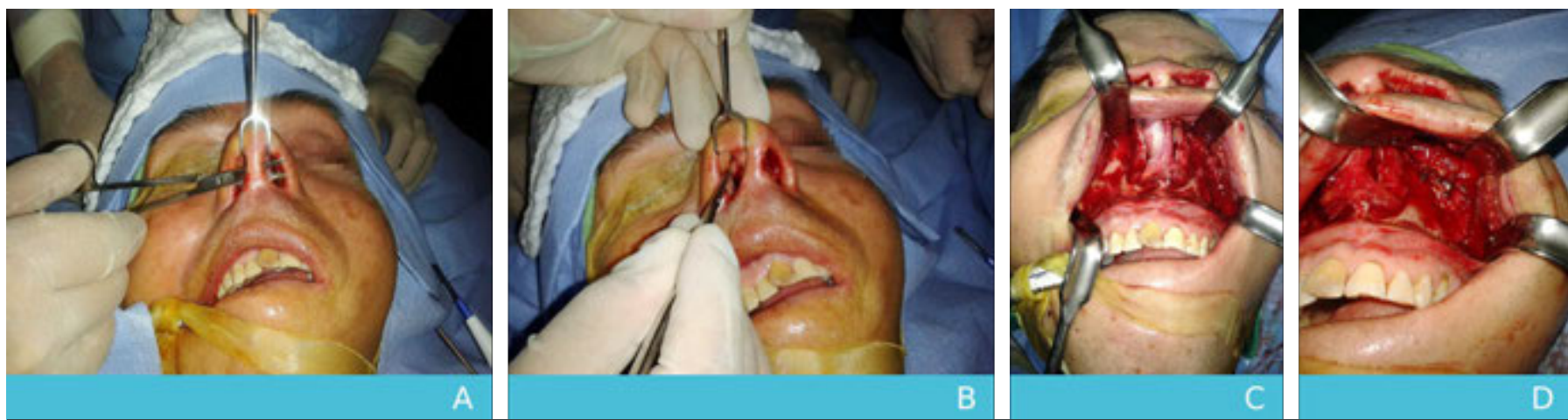

FIGURA 2. A -B: INCISIONES INTRANASALES (REALIZADAS POR EL OTORRINOLARINGÓLOGO). C: DISECCIÓN Y LEVANTAMIENTO DE COLGAJO. D: EXPOSICIÓN DE CAMPO OPERATORIO.

Este abordaje permitió una amplia exposición рага el retiro de material de osteosíntesis y corrección de la secuela de trauma facial. Figura 2.

\section{Discusión}

Existen diversas patologías en el tercio medio facial, el cirujano siempre intenta conseguir su resolución completa, pero se encuentra con barreras anatomo-quirúrgicas que lo limitan, lo que hace que opte por diversos abordajes como el Degloving centro facial que fue descrito por Casson y cols. en 1974 y desarrollado posteriormente en 1986 por Price. Este abordaje intraoral puede combinarse con osteotomías de Le Fort II o rinotomías uni o bilaterales y permite el acceso a toda la región centro facial de la cara. Otro abordaje es el extraoral Weber Ferguson al que se considera la base de los colgajos mucoosteo-musculo-cutáneos de importancia en la cirugía transfacial y sus modificaciones $\mathbf{5 , 6}$.

Los abordajes quirúrgicos para tercio medio en trauma maxilofacial tradicionalmente se han realizado a través de incisiones cutáneas que incluyen abordajes subciliar o blefaroplastia y subtarsal que exponen el reborde infraorbitario y piso de orbita, dejando una pequeña cicatriz imperceptible; el abordaje trasconjutival que expone reborde infraorbitario y piso de orbita, la cicatriz queda oculta en la conjuntiva y no requiere la disección de piel o musculo; el abordaje supraobitario o cola de ceja fácil de camuflar, que expone el borde supraorbitario y sutura fronto cigomatica. Así como abordajes trans orales (vestibular) que no dejan cicatriz visible y permiten la exposición del tercio medio de la cara y esqueleto desde el arco cigomático, reborde infraorbitario y apófisis fronto cigomatica. Sin embargo, la elección de los diferentes abordajes no solo se basa en la extensión y características de la lesión, sino también en las habilidades, la experiencia, capacitación del cirujano y la elección del paciente ${ }^{3,6}$.

El Deglovin es un abordaje intraoral que permite una mayor exposición del campo quirúrgico sin necesidad ningún tipo de abordaje externo, todas las incisiones permanecen ocultas en el surco gingivolabial y en el vestíbulo nasal. Este abordaje fue utilizado por primera vez en trauma facial por Baumann y Ewers, quienes lo aplicaron en 14 pacientes con fracturas del tercio medio facial, y concluyen que esta técnica permite la exposición de las estructuras del tercio medio facial sin incisiones cutáneas externas ${ }^{6}$.

El procedimiento de disección del tercio medio facial requiere de habilidad tanto en cirugía de senos paranasales como de rinoplastia básica, debido a que el abordaje involucra una incisión bilateral sublabial, junto con elevación del tejido blando de todo el dorso nasal. La irrigación de la piel desinsertada es suplida por las arterias 
infraorbitaria y facial, por lo que permite otras incisiones para una exposición adicional sin compromiso vascular de la zona ${ }^{6}$.

A través del abordaje Degloving se trata lesiones de casi todo el macizo facial, con resultados estéticos aceptables, como tumores grandes en una o ambas fosas nasales, fracturas de piso de orbita, entre otros; sin embargo, presenta limitaciones en ciertas regiones como la lámina papirácea, las celdillas etmoidales supraorbitarias, la fosa lacrimal y el seno frontal ${ }^{8}$.

Esta vía trans oral permite el trabajo a nivel de los senos maxilares, fosas nasales, suelos orbitarios, cigomas, fosas pterigopalatinas e infratemporales y nasofaringe, sin embargo, esta vía no es muy adecuada para la exposición del etmoides, ducto fronto-nasal y lámina; por lo pueden realizarse osteotomías medial, lateral y superior, retirando el proceso frontal del maxilar y huesos propios nasales ${ }^{8}$.

Según Méndez y cols. en el 2008, son raras las complicaciones del Degloving, siendo la más común estenosis del vestíbulo nasal que ocurrió aproximadamente en el $5 \%$ de los pacientes y no se ha reportado alteración del crecimiento facial en niños, rinitis atrófica, epífora o fístula oroantral. James J y cols. en el 2010, describen como la complicación más frecuente relacionada directamente con el procedimiento de Degloving a la anestesia infraorbitaria temporal o la hiperestesia; sin embargo, la mayoría de los pacientes recuperaron completamente la sensibilidad de 1-5 meses, y que la tasa de anestesia infraorbitaria permanente fue inferior al 3\%.Vass y cols en el 2017, no reportaron efectos secundarios por la técnica de Deglovin sin embargo, entre los pacientes operados con la técnica Weber Ferguson si se han producido efectos secundarios $\mathbf{7 , 9 , 1 1}$.

Estos abordajes no solo se usan para procedimientos oncológicos $\mathrm{o}$ de otorrinolaringología, en el Méndez y cols. aplicaron esta técnica de Deglovin, en trauma facial en un paciente con fractura panfacial, con la intención de proporcionar un tratamiento quirúrgico sin incisiones cutáneas ${ }^{6}$.

Según Kavin y cols. en el 2010, el abordaje Weber-Ferguson fue descrito como la incisión de elección para la exposición la órbita o el maxilar, considerando que el acceso a la parte más lateral del maxilar y fosa infratemporal puede verse limitado por el haz neurovascular infraorbitario 10.

Hernández y cols. en el 2007, consideraron que las técnicas cerradas, que no dejan cicatrices externas son menos agresivas, sin embargo, no se deben perderse las perspectivas terapéuticas en beneficio de las cosméticas ${ }^{7}$.

\section{Conclusiones}

- El abordaje Deglovin puede considerarse como un acercamiento excelente, útil, y seguro para muchas lesiones del tercio medio que tiene una tasa baja de complicación, con resultados cosméticos excelentes. Proporciona una excelente exposición de la porción media del esqueleto craneofacial y evita las incisiones externas.

- El abordaje Weber Ferguson proporciona una mejor visibilidad y acceso evitando así la resección inadvertida de las estructuras vitales no implicadas.

- El trabajo multidisciplinario, la buena planificación, la ejecución de osteotomías y el manejo cuidadoso de los tejidos blandos minimizan las complicaciones en cualquier abordaje que utilice el cirujano.

\section{Bibliografía}

1. Raspall Guillermo. Cirugía Maxilofacial. Patología quirúrgica de la cara, boca, cabeza y cuello. España: Editorial Panamericana;2002. 
2. Рarameswaran,

Anantanarayanan, Jayakumar, NaveenKumar, Ramanathan, Manikandhan, Hermann. Mid-Face Degloving: An Alternate Approach to Extended Osteotomies of the Midface. Journal of Craniofacial Surgery. 2017;28: 245-247

3. G. Rajasekhar, Nanda Gopal Vura, R. Sudhir, Srikanth Dhanala, and Aditya Mohan Alwala Versatility of Dieffenbach's Modification of Weber Fergusson's Approach for Treatment of Maxillary Pathologies. Journal of Maxillofacial Oral Surgery. 2016; 11(4): 416-419.

4. Hupp James, Ellis III Edward, Tucker Myron. Cirugía Oral y Maxilofacial contemporánea. España: Editorial Elsevier;2009.

5. Coca Pelaz A., López Álvarez F., Suárez Nieto C. Tratado de Otorrinolaringología y Cirugía de Cabeza y Cuello. Buenos Aires: Editorial Médica Panamericana;2008.

6. Méndez Tenorio, Sahagun Pille. Tratamiento de fractura del tercio medio facial mediante la técnica de Midfacial degloving. Reporte de un caso. Revista Española de Cirugía Oral y Maxilofacial.2008;30(5):371-377.
7. Hernández Altemir, Francisco, Hernández Montero, Sofía, Hernández Montero, Susana, Hernández Montero, Elena, Moros Peña, Manuel. Una versátil vía de abordaje al esqueleto del tercio medio facial. Revista Española de Cirugía Oral y Maxilofacial. 2007, 29(3), 182-187.

8. Shah Jatin. Cirugía y Oncología de Cabeza y Cuello. Madrid: Editorial Elsevier;2004.

9. James J. Jaber, Francis Ruggiero, Chad A. Zender. Facial degloving approach to the midface Operative Techniques in Otolaryngology. 2010;21 (3):171-174.

10. Kavin A. Andi, Simon B. Holmes, lain L. Hutchison. Infraorbitalorbitotomy: Modification of the Weber-Ferguson approach. British Journal of Oral and Maxillofacial Surgery.2010;48(1): 44-45.

11. Vass G, Bella Z, Tóbiás Z, Nagy A, Iván L, Rovó L. Esthetically favorable surgical alternative for the removal of sinonasal malignant tumors the modified facial degloving technique. Journal Oral Maxillofacial Surgery. 2017;75(10): 2272.e1 - 2272.e10. 